\title{
Specificity of the requirement for Foxo3 in primordial follicle activation
}

\author{
George B John, Lane J Shirley, Teresa D Gallardo and Diego H Castrillon \\ Department of Pathology, University of Texas Southwestern Medical Center, 6000 Harry Hines Boulevard, Dallas, \\ Texas 75390-9072, USA \\ Correspondence should be addressed to D H Castrillon; Email: diego.castrillon@utsouthwestern.edu
}

\begin{abstract}
Primordial follicles are long-lived structures assembled early in life. The mechanisms that control the balance between the conservation and the activation of primordial follicles are critically important for fertility and dictate the onset of menopause. The forkhead transcription factor Foxo3 serves an essential role in these processes by suppressing the growth of primordial follicles, thereby preserving them until later in life. While other factors regulating primordial follicle growth have been described, most serve multiple functions at several stages of female germ cell or follicle development, and corresponding mouse mutants exhibit pleiotropic phenotypes with disruption of multiple stages of follicle assembly, development, or survival. To investigate the possibility that Foxo3 also functions in other aspects of ovarian development beyond its known role in primordial follicle activation (PFA), we performed detailed analyses of mouse ovaries including electron microscopy to study primordial follicle structure, assembly, and early growth. These analyses revealed that the timing of primordial follicle assembly, early oocyte survival, and the expression of early germ line markers were unaffected in early Foxo3 ovaries. Taken together, these studies demonstrate that the phenotype associated with Foxo3 deficiency is remarkably specific for PFA and further support the placement of Foxo3 in a unique phenotypic class among mammalian female sterile mutants. Lastly, we discuss the implications of the specificity of this mutant phenotype with regard to the hypothesis that oocyte regeneration may occur in adults and serves as a means to replenish oocytes lost via natural physiological processes.
\end{abstract}

Reproduction (2007) 133 855-863

\section{Introduction}

Primordial follicle activation (PFA), also known as follicle initiation, is the metered process by which primordial follicles are removed from the long-lived reserve pool to enter into the growing follicle pool (Skinner 2005). Since follicle growth is irreversible (Peters et al. 1975, Lintern-Moore \& Moore 1979, McGee \& Hsueh 2000), the mechanisms which regulate PFA ensure that some number of growing follicles is available during each estrus/menstrual cycle, but at the same time serve to forestall premature depletion of primordial follicles and reproductive senescence. Spontaneous oocyte apoptosis is another major physiological mechanism that also contributes to follicle depletion, particularly during development and in the early postnatal period, but also throughout adult life (Tilly 2001). Elucidation of the molecular mechanisms that regulate PFA and early follicle growth and preserve primordial follicles are important remaining challenges in reproductive biology (Matzuk et al. 2002).
PFA, unlike later stages of follicle maturation including ovulation, appears to be regulated largely within the ovary itself, and is independent of pituitary gonadotropins, as evidenced by several observations: 1) it occurs in a subset of primordial follicles in newborn mouse ovaries explanted and maintained in vitro (Eppig \& O'Brien 1996), 2) PFA begins well before the onset of sexual maturity - in fact soon after birth in both mice and humans, and 3) PFA is not impeded by hypophysectomy, treatment with exogenous gonadotropins, or in mice bearing knockout mutations in the genes encoding follicle-stimulating hormone, luteinizing hormone, or their receptors (Peters et al. 1973, Mason et al. 1986, Elvin \& Matzuk 1998). Although apparently regulated predominantly via ovarian-intrinsic mechanisms, there is also evidence of modulation of PFA by systemic factors (Nelson et al. 1985, Fortune et al. 2000).

The molecular pathways controlling PFA remain poorly understood (Braw-Tal 2002). Several studies have implicated an interaction between kit ligand (KL), produced by granulosa cells, and its receptor kit, expressed on the 
oocyte membrane (Driancourt et al. 2000). Genetic evidence for this comes from studies of mutants of the Steel locus encoding KL. Female mice homozygous for the hypomorphic allele Steel panda form ovaries with an apparent primordial follicle growth arrest (i.e., failure to undergo PFA; Huang et al. 1993, Bedell et al. 1995). Other combinations of Steel alleles have similar phenotypic consequences (Kuroda et al. 1988). However, these mutations also result in pleiotropic effects due to the diverse roles of kit signaling in germ cells and result in ovaries that are severely atrophic with very few primordial follicles, clouding interpretation of these phenotypes. In vitro studies have also implicated kit and KL in PFA (Packer et al. 1994, Yoshida et al. 1997, Parrott \& Skinner 1999, Reynaud etal. 2000). Nonetheless, a clear picture of how $\mathrm{KL}$ triggers PFA has not emerged, or if indeed $\mathrm{KL}$ is necessary and sufficient for this process. Other growth signals may serve such a role (Skinner 2005).

The forkhead transcription factor Foxo3 has a specific and essential role in PFA. Foxo3 is dispensable for embryonic development, but primordial follicles in Foxo $3^{-1-}$ ovaries undergo global activation within a few days of birth, resulting in a characteristic syndrome of ovarian hyperplasia and early follicle depletion with consequent premature ovarian failure/secondary infertility (Castrillon et al. 2003, Hosaka et al. 2004). Remarkably, a distantly related forkhead transcription factor, Foxl2, is also involved in follicle growth. Mutation of a single allele of the human FOXL2 gene (haploinsufficiency) results in the autosomal dominant blepharophimosis, ptosis, epicanthus inversus syndrome (BPES) syndrome associated with eyelid anomalies and premature ovarian failure (Crisponi et al. 2001). Foxl $2^{-1-}$ female mice are sterile due to widespread follicular atresia and an apparent block in early follicle growth (Schmidt et al. 2002, Uda et al. 2004). This abnormal follicle maturation may be secondary to earlier defects, since primordial follicle individualization (also known as cyst breakdown) is disrupted in Foxl2 $2^{-1-}$ ovaries (Uda et al. 2004). Thus, Foxl2 acts earlier in follicle development than Foxo3 and may serve distinct functions at multiple stages of folliculogenesis (Ottolenghi et al. 2005). Here, we present a more detailed phenotypic characterization of the ovarian defects associated with Foxo3 lack of function, with the aim of more precisely delineating the stage(s) at which Foxo3 functions in the ovary, and an eye to the question of whether Foxo3 indeed functions specifically in PFA or serves additional functions in follicle assembly or maturation.

\section{Materials and Methods}

\section{Mouse husbandry}

The Foxo3 null allele was generated and backcrossed to FVB for six generations as previously described (Castrillon et al. 2003). For breeding assays, mating pairs consisting of 21-day-old experimental $\mathrm{FoxO}^{-1-}$ or
$\mathrm{FoxO}^{+/+}$sibling control females derived from heterozygous intercrosses (Castrillon et al. 2003) and FVB stud males were placed in cages (one pair per cage) and inspected every morning. Tail genomic DNA was prepared using a Puregene DNA isolation kit (Gentra Systems, Minneapolis, MN, USA) and mice were genotyped for Foxo3 alleles using a multiplex PCR (primers available upon request). Animals were fed ad libitum on standard chow with standard light cycling conditions. All mice were housed in a pathogen-free animal facility in microisolator cages and experiments were conducted with the approval of the UT Southwestern Institutional Animal Care and Use Committee.

\section{Electron microscopy}

Ovaries were fixed in $2 \%$ glutaraldehyde $+0.1 \mathrm{M}$ sodium cacodylate and post fixed in $1 \% \mathrm{OsO}_{4}$ and $2 \%$ uranyl acetate. The samples were then dehydrated and rinsed with propylene oxide and embedded in Epon (EMbed-812, DDSA, NMA, and DMP30) and polymerized overnight in a $60^{\circ} \mathrm{C}$ oven. Sections of 50-70 nm were obtained using a Reichert ultracut $\mathrm{E}$ microtome and mounted on formvarcarbon coated copper grids. Images were obtained on a JEOL 1200 EX transmission electron microscope.

\section{Immunolocalization and TUNEL studies}

Ovaries were immediately placed in $10 \%$ buffered formalin and fixed overnight, paraffin-embedded, and cut into $5 \mu \mathrm{m}$ sections. At least ten serial sections from four different ovaries were immunostained and evaluated for each time point and each antibody. Antibodies used were laminin Ab-1 (rabbit polyclonal, catalog \# RB-082 - A0) purchased from Neo Markers/Lab Vision Corporation (Fremont, CA, USA) and an anti-mouse vasa rabbit polyclonal, courtesy of T Noce (Tanaka et al. 2000). The laminin antibody was used at 1:1000 dilution; antigen retrieval was performed at $37^{\circ} \mathrm{C}$ in pepsin for 5-10 min. The vasa antibody was used at 1:10 000 dilution; antigen retrieval was performed at $95-100{ }^{\circ} \mathrm{C}$ in $100 \mathrm{mM}$ sodium citrate buffer ( $\mathrm{pH} \mathrm{6.0)}$ for 15 min with 20 min of cooling. The detection system employed was Impress anti-rabbit IG (cat. no. MP-7401) from Vector Laboratories (Burlingame, CA, USA) with diaminobenzidine as the substratechromogen. Slides were counterstained with hematoxylin. TUNEL was performed with the Apoptag kit from Chemicon International (Temecula, CA, USA) as per the manufacturer's instructions.

\section{Results}

\section{Normal timing of primordial follicle assembly in Foxo3 mice}

We had previously shown that nullizygosity for Foxo3 results in secondary infertility due to global PFA soon 
after birth (Castrillon et al. 2003). To study the progress of primordial follicle assembly and individualization in greater detail, we performed immunolocalization of the germ cell marker vasa. Prior to individualization, oogonia form syncytial clusters, sharing a common cytoplasm linked by intercellular bridges. During the process of individualization, the specialized stromal cells that will become pregranulosa cells (PGs) 'invade' these clusters, extending intercellular processes to physically separate the oogonia, a process known as programmed cyst breakdown. Vasa localization in the cytoplasm is continuous in syncytial clusters but becomes interrupted following individualization because of the interpolation of these somatic cell processes. Immunostaining for vasa is thus a useful tool to track the individualization of primordial follicles (Pepling \& Spradling 2001, Jefferson et al. 2006).

At postnatal day (PND) 1, most vasa-positive cells in wild-type control ovaries were associated together in syncytial clusters of two or more cells, sharing a contiguous vasa-positive cytoplasm (Fig. 1A). By PND3, individualization was largely complete, although occasional clusters remained. In contrast, by PND7, individualization was essentially complete, and even closely apposed oocytes at PND7 and PND14 were clearly separated by a distinct rim of vasa-negative cytoplasm. In PND1-14 Foxo3 $^{-1-}$ ovaries, the timing and completion of primordial follicle individualization as assessed by vasa immunostaining were unaffected, proceeding in an identical fashion as observed in the wild-type controls (Fig. 1A).

To confirm these findings, we also studied the expression of laminin, an abundant component of the basement membrane surrounding ovarian follicles. This basement membrane undergoes extensive reorganization during primordial follicle individualization and subsequent follicle growth (Lee et al. 1996). In both primordial and growing follicles, laminin is localized to
A
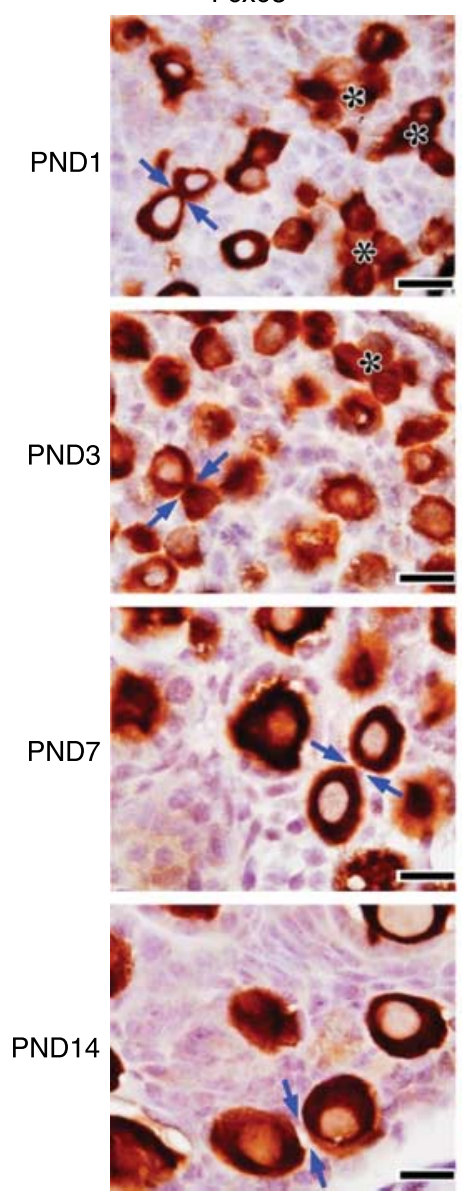

Foxo3-1-
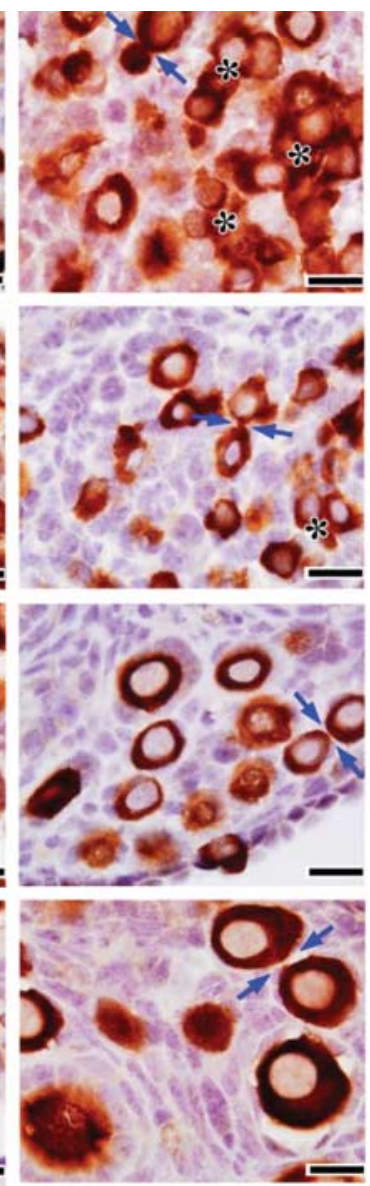

B
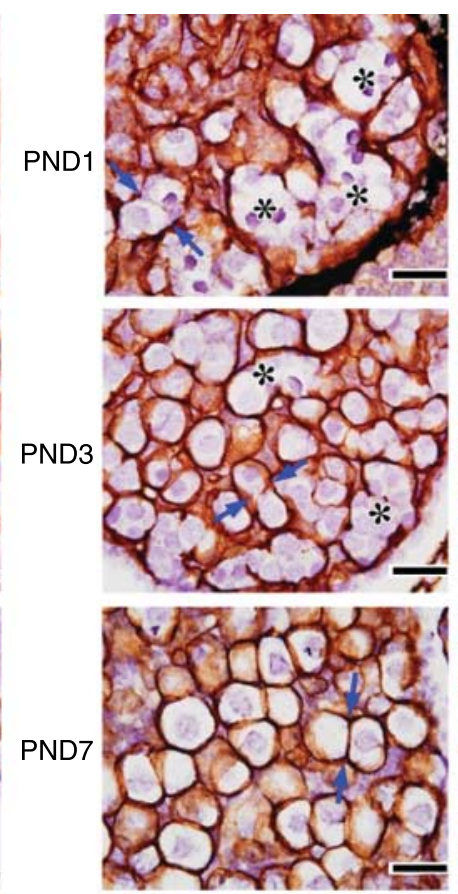

Foxo3 $3^{+/+}$
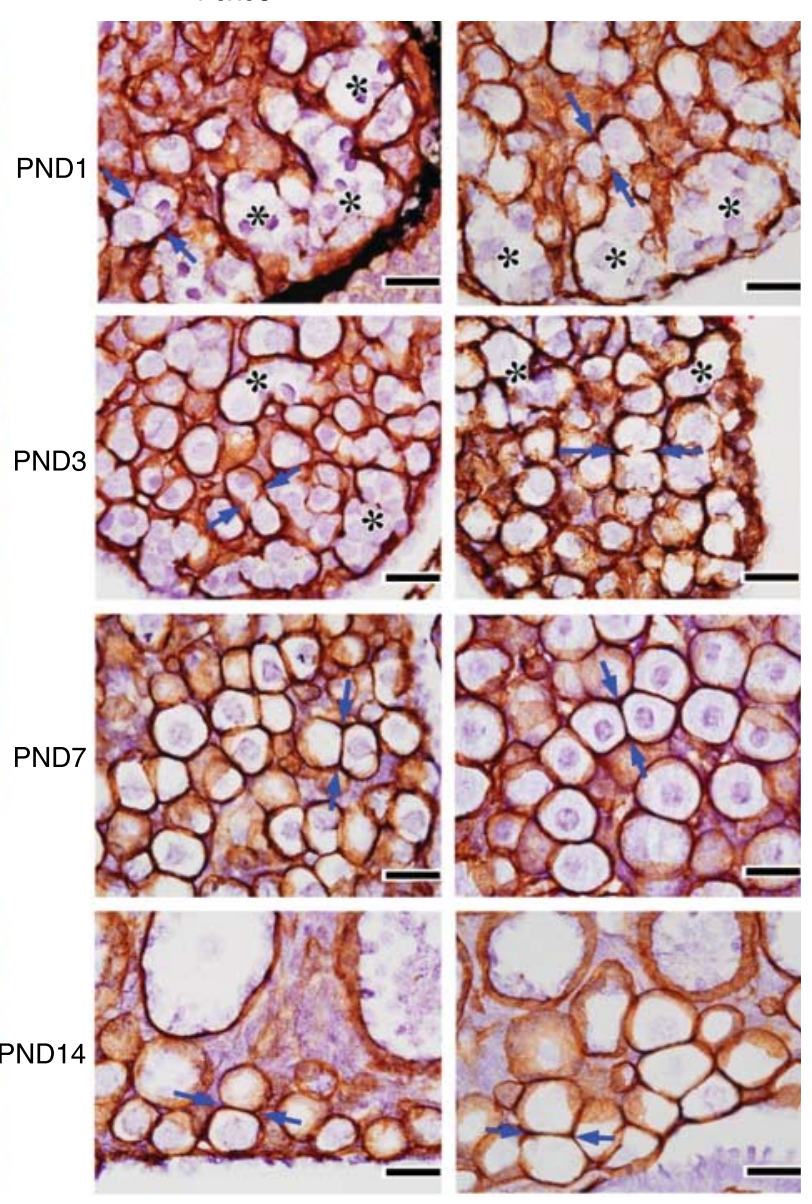

Figure 1 Programmed cyst breakdown in mouse ovaries is unaffected by Foxo3 deficiency. (A) Immunohistochemistry against vasa shows shared cytoplasm in syncytial germ cell clusters prior to individualization/cyst breakdown. (B) Immunohistochemistry against laminin shows basement membrane surrounding either germ cysts or fully individualized follicles. These studies revealed that the timing of cyst breakdown (arrows) is identical in $\mathrm{FOxO}^{+/+}$and Foxo3 ${ }^{-/-}$ovaries and is completed by PND7 in both genotypes. For A and B, arrows point to cysts in the process of breakdown (PND1 and 7) and to fully individualized follicles (PND7 and 14). Asterisks identify germ cell clusters. Bars $=20 \mu \mathrm{m}$ for all panels. 
the external aspect of the outermost layer of granulosa cells, making it a useful marker to delineate both germinal cysts and individualized follicles. Consistent with the results obtained with vasa, laminin immunohistochemistry at PND1-PND14 showed essentially complete individualization by PND7, with no delay or abnormality in Foxo ${ }^{-1-}$ ovaries relative to controls.

Polyovular follicles, abnormal follicles with more than one oocyte, are abundant in the setting of exposure to specific teratogens (geninstein and diethylstilbestrol; Iguchi et al. 1990, Jefferson et al. 2002) and in some mutants affecting follicle development (e.g. Bmp15; Yan et al. 2001), and presumably arise through defects in programmed cyst breakdown or other aspects of folliculogenesis. We note that such polyovular follicles were undetectable in serially sectioned $\mathrm{Foxo}^{-1-}$ ovaries (data not shown).

To determine if deletion of Foxo3 affects the number of syncytial clusters when compared with wild-type controls, we performed quantitation in serially sectioned, vasa immunostained PND1 ovaries ( $N=3$ per genotype). There was no significant difference in the mean number of syncytial clusters between the Foxo $3^{+/+}(41 \pm 6.1$ (s.E.M.)) and FoxO $^{-1-}(45 \pm 6.2$ (s.E.M.) $)$ ovaries at PND1.

To test whether the oocyte enlargement in $\mathrm{Foxo3}^{-1-}$ ovaries resulted in a concomitant increase in the number of somatic cells surrounding each oocyte, we performed a quantitative assessment of the mean number of somatic cells surrounding oocytes in $\mathrm{FOxO}^{+/+}$and $\mathrm{FoxO}^{-1-}$ ovaries at PND7 and PND14 ( $N=3$ per genotype). A total of 50 primordial follicles were counted from each ovary. While there was no significant increase in the mean number of somatic cells surrounding the oocyte at PND7 $\left(\right.$ FoxO $^{+/+}=3.76 \pm 0.08$ (s.E.M.), FoxO3 ${ }^{-/-}=3.86 \pm 0.12$ (S.E.M.)), we observed a relatively minor increase at PND14 $\left(\right.$ Foxo $^{+/+}=3.98 \pm 0.10$ (s.E.M.), Foxo $^{-1-}=$ $4.4 \pm 0.11$ (S.E.M.)). This finding is consistent with the absence of mitotic activity in follicles that have not undergone a transition to cuboidal granulosa cells. We conclude on the basis of these studies that Foxo3 is not required for the timing or other aspects of follicle individualization/cyst breakdown.

\section{Ultrastructural analyses: Foxo3 deficiency does not affect primordial follicle assembly or subcellular ultrastructure}

Ovarian follicles are highly complex and undergo intricate structural changes during their formation and subsequent development (Wassarman \& Josefowicz 1978). Since global PFA in Foxo3 ${ }^{-1-}$ ovaries closely coincides with the completion of follicle individualization (Castrillon et al. 2003), we considered the possibility that $\mathrm{FOxO}^{-1-}$ primordial follicles may be structurally abnormal and that such abnormalities might correlate with or be a direct cause of the global activation phenotype. To determine whether there were any such structural abnormalities in primordial follicles, we studied $\mathrm{FOXO}^{+/+}$and Foxo3 ${ }^{-/-}$ovaries at PND1, 3, 7 , and $14(N=3$ females per genotype, a total of 24 samples). These time points correspond to follicle development prior to individualization (PND1); complete assembly/individualization and the earliest manifestation of the Foxo3 ${ }^{-1-}$ phenotype (PND3-7); and early follicle growth (PND14).

Consistent with the vasa and laminin immunolocalization studies, all aspects of follicle assembly appeared unaffected in the mutant ovaries, resulting in fully individualized primordial follicles consisting of ultrastructurally normal oocytes surrounded with squamoid PGs by PND7 (Fig. 2A and B). We also compared a number of ultrastructural features and organelles at each time point as discussed below; representative images are shown for PND7 (Fig. 2).

In resting primordial follicles, the interface between oocytes and PGs consists of an elaborate complex of interdigitating microvilli with occasional gap-junctional contacts. This interface, believed to facilitate direct communication and nutrient exchange between oocytes and granulosa cells (Anderson \& Albertini 1976), was structurally unaltered in Foxo ${ }^{-1-}$ ovaries (Fig. 2C and D). We also did not observe differences in the morphology, distribution, or number of gap junctions between oocytes and PGs (not shown). Mitochondria, which are numerous in oocytes and likely serve multiple functions in metabolism and apoptosis (Perez et al. 1999, Perez et al. 2000, Tilly 2001), also were unaltered in Foxo3 ${ }^{-1-}$ oocytes and exhibited an identical pattern of tubular cristae and often a single vacuole at PND7 (Fig. 2E and F). Large multilamellar golgi networks with bud-like extensions and multiple endocytic vesicles, which are abundant in the ooplasm, were also unaltered (Fig. 2G and H). We failed to observe differences in the morphology of other organelles or substructures including the nucleus and nucleolus, ribosomes, or smooth endoplasmic reticulum, either in oocytes or granulosa cells.

At PND7 and PND14, Foxo3 ${ }^{-1-}$ oocytes appeared enlarged relative to controls, consistent with growth secondary to global follicle activation by this time point as previously documented by histomorphometry (Castrillon et al. 2003). In summary, we conclude that $\mathrm{FoxO}^{-1-}$ primordial follicles are morphologically normal and that the $\mathrm{FoxO}^{-1-}$ phenotype is not associated with distinct morphologic abnormalities preceding its onset.

\section{Foxo3 lack of function does not affect oocyte apoptosis}

Programmed cell death is an important physiological mechanism that reduces oocyte numbers both during embryonic development and in postnatal life (Tilly 2001). Primordial follicle individualization coincides with a high rate of oocyte apoptosis, and it has been 
Foxo3 +/+
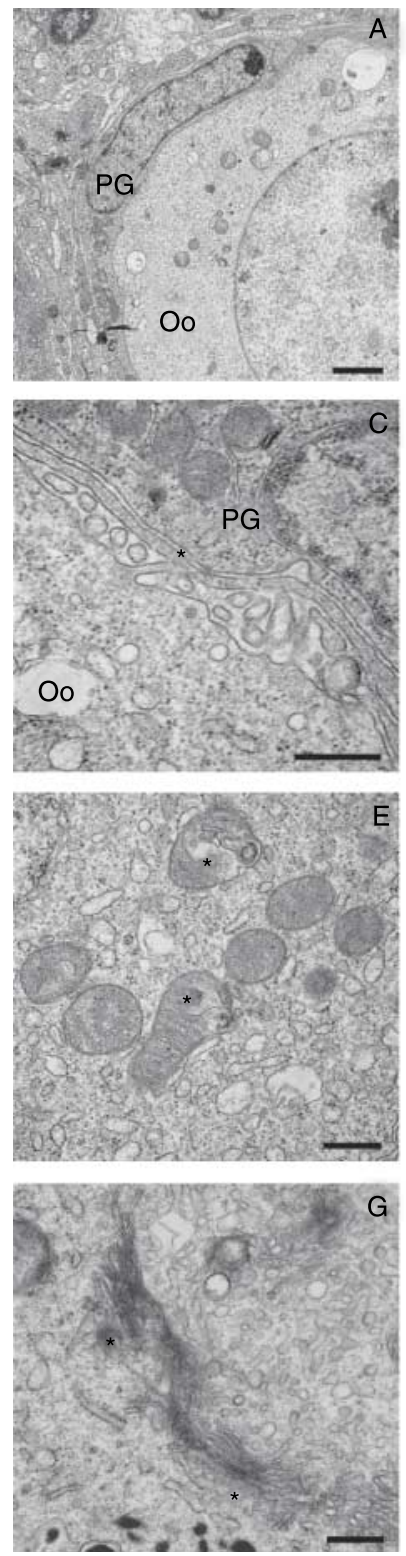

Figure 2 Foxo3 deficiency does not result in primordial follicle ultrastructural defects, representative electron microscopic images from PND7 FoxO3 ${ }^{+/+}$and Foxo3 ${ }^{-1-}$ sibling females. (A and B) Low magnification showing portion of individual primordial follicles including a single pregranulosa cell (PG) and oocyte (Oo). (C and D) High magnification of interface between oocyte and pregranulosa cell; asterisk indicates interdigitating microvilli at interface. (E and F)

Mitochondria with identical patterns of cristae and characteristic single large vacuoles (asterisks). ( $\mathrm{G}$ and $\mathrm{H}$ ) Large golgi complexes (asterisks) that occur in large numbers in the ooplasm. Bar $=2 \mu \mathrm{m}(\mathrm{A}$ and $\mathrm{B})$ or $500 \mathrm{~nm}(\mathrm{C}-\mathrm{H})$.

proposed that apoptosis contributes to the process of cyst breakdown (Pepling \& Spradling 2001). Numerous studies link the Foxos, including Foxo3, to the regulation of cell death via transcriptional control of apoptotic factors such as Bim \& FasL (Brunet et al. 1999, Moller et al. 2005) among others, prompting us to consider the possibility that Foxo3-deficient ovaries might exhibit defects in oocyte apoptosis in addition to the observed defects in PFA. TUNEL analysis performed on serial sections at PND3 ( $N=4$ ovaries per genotype), revealed no differences either in the overall pattern of TUNEL positive oocytes (Fig. 3A and B) or in the apoptotic index of oocytes in FoxO3 ${ }^{+/+}$and FoxO3 ${ }^{-/-}$ovaries (Fig. 3C); we also did not observe significant differences at PND1 or 7 (not shown).

\section{Foxo3 lack of function does not impair the progress of follicle maturation}

Foxo $^{-1-}$ females have viable litters prior to total follicle depletion at $\sim 15$ weeks of age, demonstrating that subsequent steps of follicle maturation following PFA are not completely disrupted by Foxo3 lack of function. In an attempt to uncover subtle defects or delays in follicle maturation or other processes, we determined the earliest age at which females could give birth in matings of virgin females starting at 3 weeks of age. This serves as an indirect measurement of several aspects of reproductive function, including sexual maturation, follicle growth, and gestation. The minimum time interval required to complete the process of follicle maturation (from PFA to ovulation) is difficult to measure directly, but has been estimated at 3-5 weeks (Oakberg 1979, Hirshfield 1991), consistent with the fact that the first waves of follicle activation normally begin soon after birth and that female mice normally begin to ovulate at 4-5 weeks of age. Thus, in the mouse, in contrast to longer-lived mammals, the onset of ovulation/sexual maturity coincides closely with the minimum time required for follicle maturation. The age at first litter in $\mathrm{FoxO}^{-/-}$closely matched that of $\mathrm{FoxO}^{-/+}$or FoxO $^{+/+}$sibling controls (Fig. 4A), arguing against a significant delay or lag in follicle maturation. We do note, however, that granulosa cells in only a subset of follicles assume a cuboidal morphology and grow in the Foxo $3^{-1-}$ ovaries, demonstrating that oocyte and granulosa cell growth are not completely coupled in Foxo3 $^{-1-}$ ovaries.

To study the progression of follicle maturation in more detail, we analyzed a number of markers known to be turned on or differentially expressed at different stages of follicle maturation. Inhibin is undetectable in primordial follicles and is first expressed in the granulosa cells of primary follicles. p70 S6 kinase, which phosphorylates the S6 protein of the $40 \mathrm{~S}$ ribosomal subunit and is involved in translational control of $5^{\prime}$-oligopyrimidine tract mRNAs (Manning 2004), is phosphorylated during the transition from primary to secondary follicles, presumably serving to promote the protein synthesis required for subsequent oocyte growth (unpublished data). Connexin-43, which is required for gap-junction formation and communication between granulosa cells 

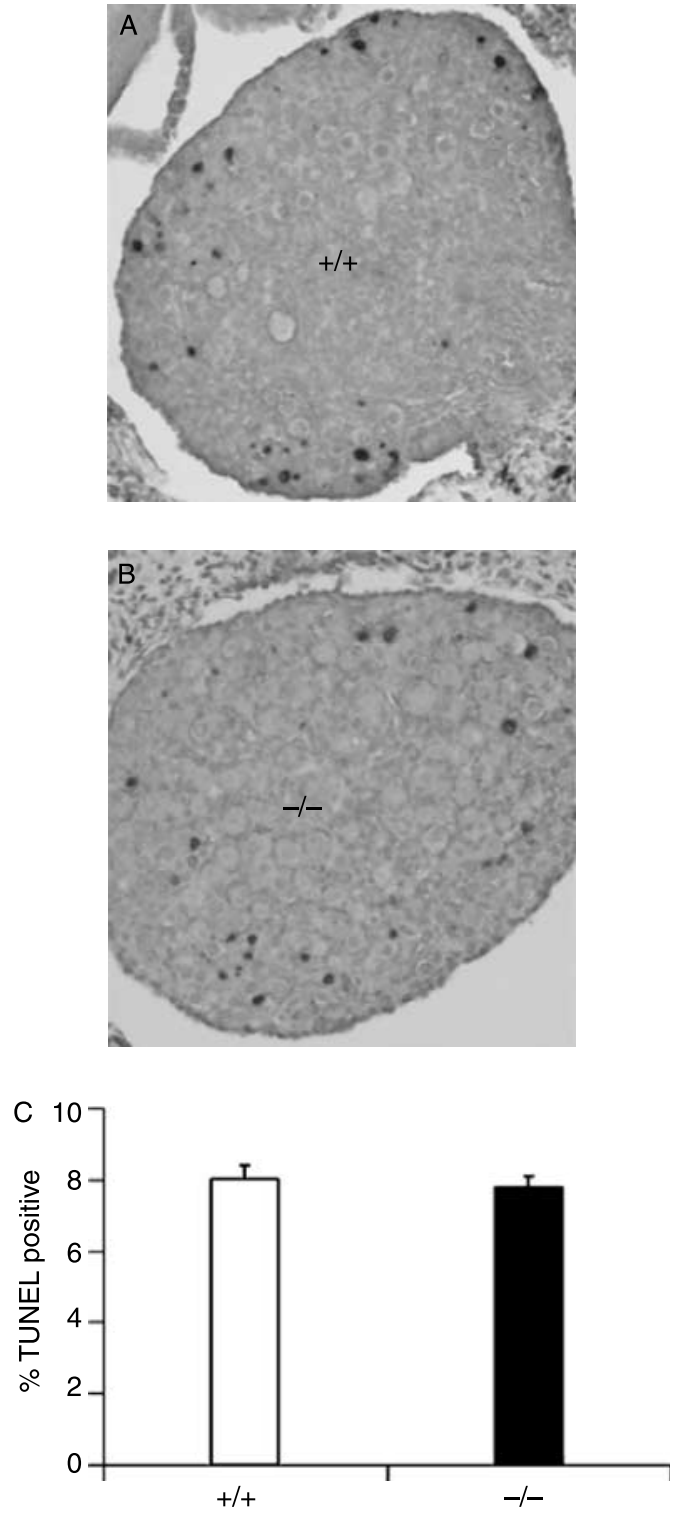

Figure 3 Oocyte apoptosis is unaffected by Foxo3 deficiency. TUNEL staining was performed on ovarian tissue sections from sibling female mice at PND3. (A) Control Foxo3 $3^{+/+}$ovary. (B) Foxo3 ${ }^{-1-}$ ovary. Apoptotic oocytes are observed in characteristic cortical pattern similar in both genotypes. (C) Apoptotic index (percentage of TUNEL positive oocytes) in Foxo3 ${ }^{+/+}$versus Foxo3 ${ }^{-/-}$ovaries at PND3; data are the mean from $N=3$ females per genotype. Error bars represent the S.E.M.; at least 500 oocytes were counted per ovary.

(Ackert et al. 2001, Teilmann 2005), is first detectable in primary follicles, and its levels continue to increase during the progression to the secondary and antral stages. For all of these markers, the timing of these changes in expression was identical, stage-for-stage, in $\mathrm{FOXO}^{+/+}$and $\mathrm{FOXO3}^{-/-}$ovaries (Fig. 4B). We also did not observe stage-for-stage alterations in the expression of other markers of follicular differentiation such as $\mathrm{p} 27^{\mathrm{KIP} 1}$ or WT1 (data not shown).

\section{Discussion}

These studies demonstrate that the requirement for Foxo3 in ovarian function is remarkably specific for PFA. We were unable to detect abnormalities in cyst breakdown/follicle individualization, spontaneous oocyte apoptosis, primordial follicle ultrastructure, or in the timing of expression of representative markers of follicle maturation. These findings further support the placement of Foxo3 in a unique phenotypic class among female sterile mutants, and highlight the importance of Foxo3 in the regulation of PFA.

It is not known whether Foxo3 serves a similar, highly specific function in PFA suppression in other mammals. If such a function were conserved in humans, it is interesting to speculate that mutation or naturally occurring sequence variation at the $\mathrm{FOXO} 3$ locus might account for some cases of premature ovarian failure (menopause prior to the age of 40) or idiopathic primary amenorrhea, two female infertility syndromes associated with premature depletion of primordial follicles (Nakano et al. 1982, Anasti 1998). Despite evidence that these conditions have a hereditary component, the relevant hereditary factors remain to be identified. Extrapolating from the mouse phenotype, homozygous FOXO3 complete loss of function mutations should result in follicle depletion prior to puberty (primary amenorrhea), given the prolonged interval between birth and sexual maturity in girls. Another (not mutually exclusive) hypothesis is that heterozygosity for $\mathrm{FOXO} 3$ could result in a more subtle increase in PFA, culminating over many years or decades in premature ovarian failure. This possibility is suggested by the finding that many forkhead transcription factors are haploinsufficient, resulting in clinical disease following mutation of a single allele, as is the case for FOXL2 in the BPES premature ovarian failure syndrome (Crisponi et al. 2001, Erickson 2001). Nonetheless, such FOXO3 mutations have not yet been identified (Watkins et al. 2006).

This and our previous studies of the ovarian defects associated with Foxo3 deficiency support the following model, shown in Fig. 5. Early steps of germ cell development, including migration, and gonadal colonization are unaffected by Foxo3 lack of function. This has not been assessed directly, but can be inferred from the observation that testis size and spermatogenesis are normal in Foxo3 ${ }^{-1-}$ males, and the finding that primordial follicle counts are also normal in $\mathrm{FOxO3}^{-1-}$ females at birth (Castrillon et al. 2003). Oogenesis, including cyst formation, cyst breakdown, and follicle individualization proceed normally in $\mathrm{FOxO}^{-1-}$ ovaries (this study). Immediately after follicle individualization is completed by PND7, global PFA is triggered in $\mathrm{FoxO}^{-/-}$females, leading to increased numbers of follicles progressing through subsequent stages of follicle maturation. Despite global oocyte growth, granulosa 


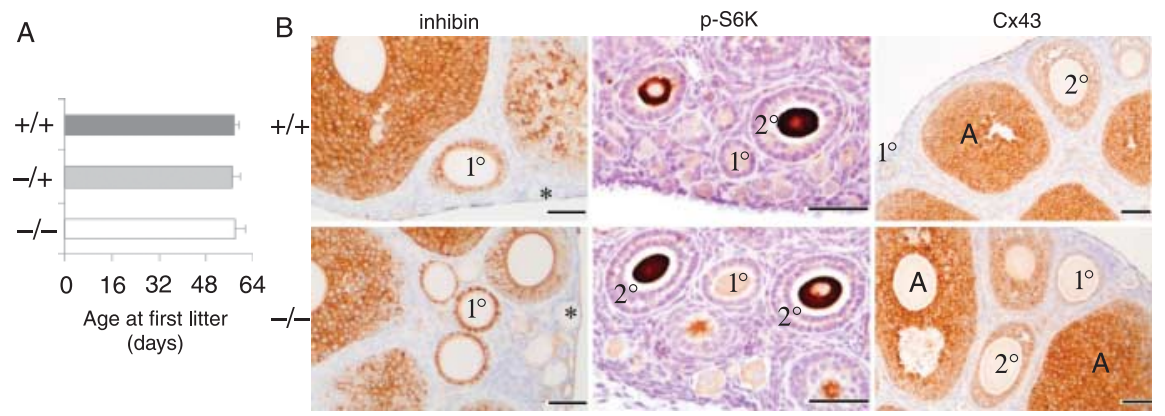

Figure 4 Evidence against constitutional defects in the progression of follicle maturation subsequent to global follicle activation in $\mathrm{FOxO}^{-/-}$ovaries. (A) Maternal age at the time of first litter. Females at 3 weeks of age (prior to sexual maturity) were placed in cages with stud males. Numbers of females analyzed were Foxo $3^{+/+}(N=5)$; Foxo $3^{-1+}(N=4)$; Foxo $3^{-1-}(N=4)$. There was no lag in Foxo3 ${ }^{-1-}$ females relative to Foxo3 ${ }^{+/+}$ and $\mathrm{FOxO}^{-1+}$ controls, arguing against a significant disruption in all subsequent stages of follicle maturation. Error bars represent the s.E.M. (B) Immunolocalization of ovarian markers at 4-5 weeks of age that characterize transitions to more advanced follicle stages, including inhibin (undetectable in primordial follicles (asterisks) and first expressed in primary follicles), S6 kinase (which becomes phosphorylated during the transition from primary to secondary follicles), and connexin-43, which is expressed at low levels in secondary follicles but becomes strongly induced by the antral follicle stage. The timing of expression of these markers in $\mathrm{FoxO}^{-/-}$ovaries closely match that observed in Foxo ${ }^{+/+} \mathrm{controls}^{-}$ Bars $=50 \mu \mathrm{m}$ for all panels.

cells in only a subset of follicles transition to cuboidal morphology and begin to proliferate. Foxo ${ }^{-1-}$ follicles appear to either undergo essentially normal growth or exhibit the abnormal pattern of continued oocyte growth in the absence of granulosa cell growth, in a rather 'all or none' manner, implying that oocyte and granulosa cell growth are only partially coupled in Foxo3 ${ }^{-1-}$ ovaries. We hypothesize that in the normal ovary, there are repressive feedback mechanisms which restrict early follicle growth and which operate normally in $\mathrm{FoxO}^{-1-}$ ovaries despite global PFA.

Increased PFA also results in striking ovarian hypertrophy by PND14 (due to a vast increase in the number of growing follicles). Foxo $3^{-1-}$ females initially have normal litters despite global PFA, but undergo a rapid decrease in fecundity that correlates with massive follicle atresia secondary to global PFA. By 15 weeks,
Foxo $^{-1-}$ ovaries are completely devoid of follicles and sterility ensues. We stress that Foxo3 ${ }^{-1-}$ females have normal progeny until the time that follicle depletion is total, indicating that other aspects of oogenesis including meiosis and all stages of follicle maturation subsequent to PFA including ovulation are unaffected. Furthermore, progeny are viable, demonstrating that 1) $\mathrm{Foxo3}^{-1-}$ oocytes support normal embryogenesis and 2) Foxo3 serves no essential function in other aspects of female reproductive function including placentation, parturition, lactation, etc.

Lastly, the exceptional phenotype associated with Foxo3 mutation presents a unique opportunity to reflect upon recent reports that de novo oocyte formation occurs in the adult mouse ovary, where evidence has been presented that a continuous influx of germline stem cells into the adult gonad replenishes oocytes lost by

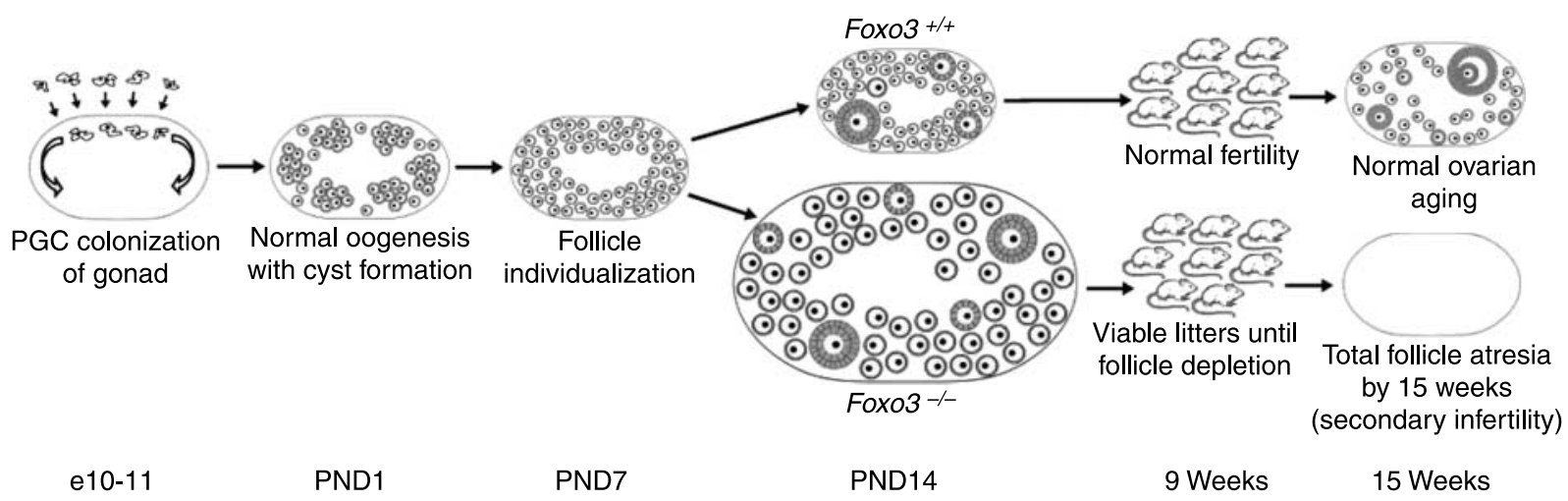

Figure 5 Schematic illustrating the Foxo3 null phenotype. Colonization of the gonad by germ line stem cells occurs normally during embryonic development (Castrillon et al. 2003), as do subsequent stages of female germline development up to cyst breakdown leading to the formation of primordial follicles (this study). Immediately following primordial follicle individualization (within a few days of birth), all primordial follicles in Foxo3 $^{-1-}$ ovaries undergo activation in a synchronized manner, resulting in dramatically increased ovarian size by PND14. Despite this global activation, maturation of some follicles proceeds normally and females have viable progeny until total follicle atresia occurs by 15 weeks. Since follicle growth is irreversible and activated, growing follicles have a finite lifespan (Elvin \& Matzuk 1998, McGee \& Hsueh 2000) and Foxo3 deficiency results in premature ovarian failure due to depletion of the follicle pool. 
normal physiological mechanisms such as apoptosis and PFA (Johnson et al. 2004, 2005), although recent studies have provided evidence against this hypothesis (Byskov et al. 2005, Eggan et al. 2006). Furthermore, the $\mathrm{FoxO}^{-1-}$ phenotype would appear to be inconsistent with such a model. If de novo primordial follicle synthesis did occur in the adult ovary, such newly synthesized follicles in Foxo $3^{-1-}$ ovaries should undergo activation at the time of their assembly, leading to ovaries with follicles at different stages of maturation (albeit possibly in reduced numbers) and female infertility would thus not be anticipated from a pure defect in the repression of PFA. However, infertility in $\mathrm{FOXO}^{-/-}$females occurs by 15 weeks of age and we do not observe any evidence of de novo follicle regeneration. Foxo ${ }^{-1-}$ ovaries after 15 weeks are always completely devoid of follicles (Fig. 5; Castrillon et al. 2003). On the other hand, we cannot exclude the possibility that Foxo3 is required in the bone marrow for the survival or function of putative germline stem cells or in the somatic ovary to sustain them. Nonetheless, it is not clear why such defects would be manifest only in adult life, since as discussed above, it is apparent from the $\mathrm{FoxO}^{-1-}$ phenotype that Foxo3 is not required for early germline or gonadal development, including the initial colonization of the gonad by germline stem cells.

\section{Acknowledgements}

We thank Toshiaki Noce for the generous gift of the anti-mouse vasa antibody. We also thank Tom Januszewski and George Lawton for help in processing and sectioning of tisssues for electron microscopic analysis. This work was supported by an $\mathrm{NIH}$ grant (HD049680) to DHC. The authors declare that there is no conflict of interest that would prejudice the impartiality of this scientific work.

\section{References}

Ackert CL, Gittens JE, O'Brien MJ, Eppig JJ \& Kidder GM 2001 Intercellular communication via connexin43 gap junctions is required for ovarian folliculogenesis in the mouse. Developmental Biology 233 258-270.

Anasti JN 1998 Premature ovarian failure: an update. Fertility and Sterility 70 1-15.

Anderson E \& Albertini DF 1976 Gap junctions between the oocyte and companion follicle cells in the mammalian ovary. Journal of Cell Biology 71 680-686.

Bedell MA, Brannan CI, Evans EP, Copeland NG, Jenkins NA \& Donovan PJ 1995 DNA rearrangements located over $100 \mathrm{~kb} 5$ ' of the Steel $(\mathrm{Sl}$ )-coding region in Steel-panda and Steel-contrasted mice deregulate $\mathrm{SI}$ expression and cause female sterility by disrupting ovarian follicle development. Genes \& Development 9 455-470.

Braw-Tal R 2002 The initiation of follicle growth: the oocyte or the somatic cells? Molecular and Cellular Endocrinology 187 11-18.

Brunet A, Bonni A, Zigmond MJ, Lin MZ, Juo P, Hu LS, Anderson MJ, Arden KC, Blenis J \& Greenberg ME 1999 Akt promotes cell survival by phosphorylating and inhibiting a forkhead transcription factor. Cell 96 857-868.
Byskov AG, Faddy MJ, Lemmen JG \& Andersen CY 2005 Eggs forever? Differentiation 73 438-446.

Castrillon DH, Miao L, Kollipara R, Horner JW \& DePinho RA 2003 Suppression of ovarian follicle activation in mice by the transcription factor Foxo3a. Science 301 215-218.

Crisponi L, Deiana M, Loi A, Chiappe F, Uda M, Amati P, Bisceglia L, Zelante L, Nagaraja R, Porcu S, et al. 2001 The putative forkhead transcription factor FOXL2 is mutated in blepharophimosis/ptosis/epicanthus inversus syndrome. Nature Genetics 27 159-166.

Driancourt MA, Reynaud K, Cortvrindt R \& Smitz J 2000 Roles of KIT and KIT LIGAND in ovarian function. Reviews of Reproduction $\mathbf{5}$ 143-152.

Eggan K, Jurga S, Gosden R, Min IM \& Wagers AJ 2006 Ovulated oocytes in adult mice derive from non-circulating germ cells. Nature 441 1109-1114.

Elvin JA \& Matzuk MM 1998 Mouse models of ovarian failure. Reviews of Reproduction 3 183-195.

Eppig JJ \& O'Brien MJ 1996 Development in vitro of mouse oocytes from primordial follicles. Biology of Reproduction 54 197-207.

Erickson RP 2001 Forkhead genes and human disease. Journal of Applied Genetics 42 211-221.

Fortune JE, Cushman RA, Wahl CM \& Kito S 2000 The primordial to primary follicle transition. Molecular and Cellular Endocrinology 163 53-60.

Hirshfield AN 1991 Development of follicles in the mammalian ovary. International Review of Cytology 124 43-101.

Hosaka T, Biggs WH III, Tieu D, Boyer AD, Varki NM, Cavenee WK \& Arden KC 2004 Disruption of forkhead transcription factor (FOXO) family members in mice reveals their functional diversification. PNAS 101 2975-2980.

Huang EJ, Manova K, Packer Al, Sanchez S, Bachvarova RF \& Besmer P 1993 The murine steel panda mutation affects kit ligand expression and growth of early ovarian follicles. Developmental Biology 157 100-109.

Iguchi T, Fukazawa Y, Uesugi Y \& Takasugi N 1990 Polyovular follicles in mouse ovaries exposed neonatally to diethylstilbestrol in vivo and in vitro. Biology of Reproduction 43 478-484.

Jefferson WN, Couse JF, Padilla-Banks E, Korach KS \& Newbold RR 2002 Neonatal exposure to genistein induces estrogen receptor (ER)alpha expression and multioocyte follicles in the maturing mouse ovary: evidence for ERbeta-mediated and nonestrogenic actions. Biology of Reproduction 67 1285-1296.

Jefferson W, Newbold R, Padilla-Banks E \& Pepling M 2006 Neonatal genistein treatment alters ovarian differentiation in the mouse: inhibition of oocyte nest breakdown and increased oocyte survival. Biology of Reproduction 74 161-168.

Johnson J, Canning J, Kaneko T, Pru JK \& Tilly JL 2004 Germline stem cells and follicular renewal in the postnatal mammalian ovary. Nature 428 145-150.

Johnson J, Bagley J, Skaznik-Wikiel M, Lee HJ, Adams GB, Niikura Y, Tschudy KS, Tilly JC, Cortes ML, Forkert R et al. 2005 Oocyte generation in adult mammalian ovaries by putative germ cells in bone marrow and peripheral blood. Cell 122 303-315.

Kuroda H, Terada N, Nakayama H, Matsumoto K \& Kitamura Y 1988 Infertility due to growth arrest of ovarian follicles in Sl/Slt mice. Developmental Biology 126 71-79.

Lee VH, Britt JH \& Dunbar BS 1996 Localization of laminin proteins during early follicular development in pig and rabbit ovaries. Journal of Reproduction and Fertility 108 115-122.

Lintern-Moore S \& Moore GP 1979 The initiation of follicle and oocyte growth in the mouse ovary. Biology of Reproduction 20 773-778.

Manning BD 2004 Balancing Akt with S6K: implications for both metabolic diseases and tumorigenesis. Journal of Cell Biology 167 399-403.

Mason AJ, Hayflick JS, Zoeller RT, Young WS III, Phillips HS, Nikolics K \& Seeburg PH 1986 A deletion truncating the gonadotropinreleasing hormone gene is responsible for hypogonadism in the hpg mouse. Science 234 1366-1371. 
Matzuk MM, Burns KH, Viveiros MM \& Eppig JJ 2002 Intercellular communication in the mammalian ovary: oocytes carry the conversation. Science 296 2178-2180.

McGee EA \& Hsueh AJ 2000 Initial and cyclic recruitment of ovarian follicles. Endocrine Reviews 21 200-214.

Moller C, Alfredsson J, Engstrom M, Wootz H, Xiang Z, Lennartsson J, Jonsson JI \& Nilsson G 2005 Stem cell factor promotes mast cell survival via inactivation of FOXO3a-mediated transcriptional induction and MEK-regulated phosphorylation of the proapoptotic protein bim. Blood 106 1330-1336.

Nakano R, Washio M, Hashiba N \& Tojo S 1982 Ovarian morphologic features and endocrine profile in amenorrheic patients. Gynecologic and Obstetric Investigation 14 19-31.

Nelson JF, Gosden RG \& Felicio LS 1985 Effect of dietary restriction on estrous cyclicity and follicular reserves in aging C57BL/6J mice. Biology of Reproduction 32 515-522.

Oakberg EF 1979 Timing of oocyte maturation in the mouse and its relevance to radiation-induced cell killing and mutational sensitivity. Mutation Research 59 39-48.

Ottolenghi C, Omari S, Garcia-Ortiz JE, Uda M, Crisponi L, Forabosco A, Pilia G \& Schlessinger D 2005 Foxl2 is required for commitment to ovary differentiation. Human Molecular Genetics 14 2053-2062.

Packer AI, Hsu YC, Besmer P \& Bachvarova RF 1994 The ligand of the c-kit receptor promotes oocyte growth. Developmental Biology 161 194-205.

Parrott JA \& Skinner MK 1999 Kit-ligand/stem cell factor induces primordial follicle development and initiates folliculogenesis. Endocrinology $1404262-4271$.

Pepling ME \& Spradling AC 2001 Mouse ovarian germ cell cysts undergo programmed breakdown to form primordial follicles. Developmental Biology 234 339-351.

Perez GI, Robles R, Knudson CM, Flaws JA, Korsmeyer SJ \& Tilly JL 1999 Prolongation of ovarian lifespan into advanced chronological age by Bax-deficiency. Nature Genetics 21 200-203.

Perez GI, Trbovich AM, Gosden RG \& Tilly JL 2000 Mitochondria and the death of oocytes. Nature 403 500-501.

Peters H, Byskov AG, Lintern-Moore S, Faber M \& Andersen M 1973 The effect of gonadotrophin on follicle growth initiation in the neonatal mouse ovary. Journal of Reproduction and Fertility 35 139-141.

Peters H, Byskov AG, Himelstein-Braw R \& Faber M 1975 Follicular growth: the basic event in the mouse and human ovary. Journal of Reproduction and Fertility 45 559-566.
Reynaud K, Cortvrindt R, Smitz J \& Driancourt MA 2000 Effects of kit ligand and anti-Kit antibody on growth of cultured mouse preantral follicles. Molecular Reproduction and Development 56 483-494.

Schmidt M, de Mattos SF, van der Horst A, Klompmaker R, Kops GJ, Lam EW, Burgering BM \& Medema RH 2002 Cell cycle inhibition by FoxO forkhead transcription factors involves downregulation of cyclin D. Molecular and Cellular Biology 22 7842-7852.

Skinner MK 2005 Regulation of primordial follicle assembly and development. Human Reproduction Update 11 461-471.

Tanaka SS, Toyooka Y, Akasu R, Katoh-Fukui Y, Nakahara Y, Suzuki R, Yokoyama M \& Noce T 2000 The mouse homolog of Drosophila vasa is required for the development of male germ cells. Genes \& Development 14 841-853.

Teilmann SC 2005 Differential expression and localisation of connexin37 and connexin-43 in follicles of different stages in the 4-week-old mouse ovary. Molecular and Cellular Endocrinology 234 27-35.

Tilly JL 2001 Commuting the death sentence: how oocytes strive to survive. Nature Reviews. Molecular Cell Biology 2 838-848.

Uda M, Ottolenghi C, Crisponi L, Garcia JE, Deiana M, Kimber W, Forabosco A, Cao A, Schlessinger D \& Pilia G 2004 Foxl2 disruption causes mouse ovarian failure by pervasive blockage of follicle development. Human Molecular Genetics 13 1171-1181.

Wassarman PM \& Josefowicz WJ 1978 Oocyte development in the mouse: an ultrastructural comparison of oocytes isolated at various stages of growth and meiotic competence. Journal of Morphology 156 209-235.

Watkins WJ, Umbers AJ, Woad KJ, Harris SE, Winship IM, Gersak K \& Shelling AN 2006 Mutational screening of FOXO3A and FOXO1A in women with premature ovarian failure. Fertility and Sterility $\mathbf{8 6}$ 1518-1521.

Yan C, Wang P, DeMayo J, DeMayo FJ, Elvin JA, Carino C, Prasad SV, Skinner SS, Dunbar BS, Dube JL et al. 2001 Synergistic roles of bone morphogenetic protein 15 and growth differentiation factor 9 in ovarian function. Molecular Endocrinology 15 854-866.

Yoshida H, Takakura N, Kataoka H, Kunisada T, Okamura H \& Nishikawa SI 1997 Stepwise requirement of c-kit tyrosine kinase in mouse ovarian follicle development. Developmental Biology 184 122-137.

Received 1 June 2006

First decision 14 July 2006

Revised manuscript received 24 January 2007

Accepted 26 January 2007 